\title{
Biophysical Studies Reveal Key Interactions between Papiliocin- Derived PapN and Lipopolysaccharide in Gram-Negative Bacteria
}

\author{
Prasannavenkatesh Durai, Yeongjoon Lee, Jieun Kim, Dasom Jeon, and Yangmee Kim*
}

Department of Bioscience and Biotechnology, Konkuk University, Seoul 05029, Republic of Korea

\author{
Received: January 19, 2018 \\ Revised: January 30, 2018 \\ Accepted: February 1, 2018 \\ First published online \\ February 13, 2018 \\ ${ }^{*}$ Corresponding author \\ Phone: +82-2-450-3421 \\ Fax: +82-2-447-5987; \\ E-mail: ymkim@konkuk.ac.kr \\ pISSN 1017-7825, eISSN 1738-8872 \\ Copyright@ 2018 by \\ The Korean Society for Microbiology \\ and Biotechnology
}

Papiliocin, isolated from the swallowtail butterfly (Papilio xuthus), is an antimicrobial peptide with high selectivity against gram-negative bacteria. We previously showed that the Nterminal helix of papiliocin (PapN) plays a key role in the antibacterial and anti-inflammatory activity of papiliocin. In this study, we measured the selectivity of PapN against multidrugresistant gram-negative bacteria, as well as its anti-inflammatory activity. Interactions between Trp2 of PapN and lipopolysaccharide (LPS), which is a major component of the outer membrane of gram-negative bacteria, were studied using the Trp fluorescence blue shift and quenching in LPS micelles. Furthermore, using circular dichroism, we investigated the interactions between PapN and LPS, showing that LPS plays critical roles in peptide folding. Our results demonstrated that Trp2 in PapN was buried deep in the negatively charged LPS, and Trp2 induced the $\alpha$-helical structure of PapN. Importantly, docking studies determined that predominant electrostatic interactions of positively charged arginine residues in PapN with phosphate head groups of LPS were key factors for binding. Similarly, hydrophobic interactions by aromatic residues of PapN with fatty acid chains in LPS were also significant for binding. These results may facilitate the development of peptide antibiotics with antiinflammatory activity.

Keywords: Antimicrobial peptide, PapN, inflammation, LPS, antibiotics

\section{Introduction}

Antimicrobial peptides (AMPs) play important roles in the innate immunity of various organisms [1, 2]. Cecropin was the first $\alpha$-helical AMP to be isolated from the hemolymph of bacteria-challenged, diapausing pupae of the giant silk moth Hyalophora cecropia. Subsequently, several cecropins with a length of 31 to 42 amino acids were identified from other insects [3,4]. These compounds are highly cationic and have conserved aromatic residues located at the N-terminus. Papiliocin is an insect cecropin with 37 residues (RWKIFKKIEKVGRNVRDGIIKAGPAV AVVGQAATVVK- $\mathrm{NH}_{2}$ ) found in the larvae of the swallowtail butterfly Papilio xuthus and exhibits potent antibacterial activity against gram-negative bacteria as well as antifungal activity [5-7]. The three-dimensional structure of papiliocin was determined using nuclear magnetic resonance (NMR) spectroscopy, which revealed an amphipathic N-terminal $\alpha$-helix, a C-terminal hydrophobic $\alpha$-helix, and a hinge in between [6]. Our previous studies showed that substitution of Trp2 with Ala in papiliocin proved that Trp2 plays a critical role in the antibacterial activity of papiliocin. The N-terminal helical domain of papiliocin (PapN; RWKIFKKIEKVGRNVRDGIIKA) possessed substantial antibacterial and anti-inflammatory activities, whereas the $\mathrm{C}$-terminal region did not show antibacterial activity $[8,9]$.

Several studies have focused on peptides with antiinflammatory activity that can act as potential candidates against sepsis, infection, and other inflammatory stimuli [10]. Importantly, many AMPs are known to interact with lipopolysaccharide (LPS), which stimulates inflammation during infection by gram-negative bacteria [11]. For example, the cathelicidin peptide LL-37 is highly effective at inhibiting LPS-induced cellular cytokine and nitric oxide (NO) released by binding directly to LPS or blocking the 
binding of LPS to LPS-binding protein [12, 13]. It has been reported that patients lacking LL-37 are more susceptible to bacterial infections [14]. Sepsis, which originates from a pathogenic infection, causes a serious LPS-mediated inflammatory response [15]. A dysfunction of the host immune defense system against pathogenic infection might be the major reason for sepsis. This has sparked a growing search for antibiotics to treat sepsis [16].

In this study, we explored the interactions between LPS and PapN, a possible source of short antibiotics. The antiinflammatory activity of PapN was investigated by examining its inhibition of $\mathrm{NO}$ and cytokine production in LPS-stimulated RAW264.7 cells. To understand the origin of the antibacterial and anti-inflammatory activities of PapN, we investigated the direct interaction between PapN and LPS using circular dichroism (CD) and tryptophan fluorescence studies. We also built a binding model of PapN and LPS using molecular docking study, further proving that PapN could be a potent anti-inflammatory agent.

\section{Materials and Methods}

\section{Peptide Synthesis}

PapN and other peptides were synthesized by solid-phase synthesis as described previously [17]. Peptides were purified with a purity higher than $95 \%$ by reversed-phase preparative high-performance liquid chromatography. We determined the molecular masses of the peptides using a matrix-assisted laserdesorption ionization-time-of-flight mass spectrometer at the Korea Basic Science Institute (Ochang, Korea).

\section{Antibacterial Activity}

The minimal inhibitory concentration (MIC) for each of the peptides against six gram-negative drug-resistant bacteria (Escherichia coli CCARM 1229, E. coli CCARM1238, Salmonella serovar Typhimurium CCARM 8003, S. serovar Typhimurium CCARM 8007, Acinetobacter baumannii CCARM12035, and A. baumannii CCARM12010) and two gram-positive drug-resistant bacteria (Staphylococcus aureus CCARM 3089 and S. aureus CCARM 3090) was determined using the broth microdilution method as described previously [17]. The lowest concentration of peptides that completely inhibited the growth of bacteria was considered as the MIC.

\section{Cytotoxicity}

Mouse fibroblast NIH3T3 cells were cultured in a $5 \% \mathrm{CO}_{2}$ atmosphere using Dulbecco's modified Eagle's medium supplemented with $10 \%$ heat-inactivated fetal bovine serum and antibiotics $(100 \mathrm{U} / \mathrm{ml}$ penicillin, $100 \mu \mathrm{g} / \mathrm{ml}$ streptomycin). The percentage of growth inhibition was evaluated using a 3-(4,5dimethylthiazol-2-yl)-2,5-diphenyltetrazolium bromide (MTT) assay for the measurement of viable cells as described previously [18]

\section{Tryptophan Fluorescence Blue Shift}

We prepared small unilamellar vesicles (SUVs) using egg yolk PC/PG (EYPC/EYPG) (7:3 (w/w)) as described previously [6]. SUVs were added to a peptide solution ( $5 \mu \mathrm{M}$ final concentration) in $10 \mathrm{mM}$ Tris buffer (pH 7.4), $0.1 \mathrm{mM}$ ethylenediamine tetraacetic acid, and $150 \mathrm{mM} \mathrm{NaCl}$. Trp2 from the peptide was excited at $280 \mathrm{~nm}$, and emission spectra were recorded at 300-400 $\mathrm{nm}$ using a 5-nm band pass filter. Blue shifts were measured also in $10 \mathrm{mM}$ sodium phosphate buffer.

\section{Quenching of Tryptophan Fluorescence by Acrylamide}

A peptide solution was added to LPS to maintain the LPS:peptide molar ratio. Excitation of Trp2 was performed at $295 \mathrm{~nm}$ instead of $280 \mathrm{~nm}$ to decrease the absorbance contributed by acrylamide [6]. Tryptophan fluorescence was quenched by titration with acrylamide, from a $4 \mathrm{M}$ stock solution, to the final concentration of $0.4 \mathrm{M}$ in the presence of LPS at a peptide:lipid molar ratio of 1:60. The quenching data were analyzed by using the Stern-Volmer plot with the equation $F 0 / F=1+K \operatorname{sv}[Q]$, where $F 0$ is the fluorescence of the peptide without acrylamide, $F$ is the fluorescence of the peptide with acrylamide, Ksv is the SternVolmer quenching constant, and $[Q]$ is the concentration of acrylamide.

\section{Nitric Oxide Production in LPS-Stimulated RAW264.7 Cells}

Mouse RAW264.7 macrophages $\left(1 \times 10^{5}\right)$ were plated in each well of 96-well culture plates and stimulated with LPS $(20 \mathrm{ng} / \mathrm{ml})$ from E. coli O111:B4 (Sigma-Aldrich, USA) in the presence or absence of peptides. Nitrite accumulation in the culture medium was used as an indicator of NO production [19]. Nitrite concentrations were calculated with a standard curve generated using $\mathrm{NaNO}_{2}$ as described previously [20].

\section{Quantification of Cytokine TNF- $\alpha$ Production}

Antibodiy for mouse tumor necrosis factor- $\alpha$ (mTNF- $\alpha$ ) was immobilized on immunoplates by incubating with $0.2-0.8 \mu \mathrm{g} / \mathrm{ml}$ solutions of antibody overnight at room temperature. Peptideinduced inhibition of TNF- $\alpha$ in LPS-stimulated RAW264.7 cells was determined as described previously [17]. Quantification of TNF- $\alpha$ production was determined by enzyme-linked immunosorbent assay (R\&D Systems, USA).

\section{Circular Dichroism}

CD experiments were performed using a J810 CD spectropolarimeter (Jasco, Japan) at 0.1-nm intervals from 190 to $250 \mathrm{~nm}$. CD spectroscopy was used to investigate the secondary structural changes of $50 \mu \mathrm{M}$ peptides following addition of $0-0.4 \mathrm{mg} / \mathrm{ml}$ LPS.

\section{Prediction of Binding Model}

For molecular docking of PapN/LPS, AutoDock Vina 
Table 1. Antibacterial activity of papiliocin and PapN against drug-resistant bacteria.

\begin{tabular}{lcccccccc}
\hline \multicolumn{1}{c}{ MIC $(\mu \mathrm{M})$} \\
\hline \multirow{2}{*}{ Peptides } & E. coli & E. coli & S. Typhimurium & S. Typhimurium & A. baumannii & A. baumannii & S. aureus & S. aureus \\
& 1229 & 1238 & $8007(\mathrm{R})$ & $8003(\mathrm{R})$ & $112035(\mathrm{R})$ & $12010(\mathrm{R})$ & $3089(\mathrm{R})$ & $3090(\mathrm{R})$ \\
\hline Papiliocin & 0.5 & 0.5 & 1 & 1 & 0.5 & 0.5 & 32 & 32 \\
PapN & 4 & 4 & 4 & 4 & 4 & 1 & 16 & 16 \\
Melittin & 4 & 4 & 4 & 4 & 4 & 2 & 2 \\
\hline
\end{tabular}

implemented in Yasara software was used [21]. The LPS structure (PDB ID: 3FXI) was used as the receptor after adding hydrogens and performing quick minimization using the dreiding forcefield in Discovery Studio 4.1 (Accelrys Software Inc., USA) [22]. The PapN structure was used as a ligand. The N-terminus of papiliocin (PDB ID: 2LA2) was used to extract the PapN structure as the ligand with all the atoms fixed except the side chains of all residues in the structure. The entire LPS was set as the binding site using the AutoGrid algorithm, and AutoDock Vina was applied for the docking process. The ligand was given 125 docking runs and we retrieved the docked conformations through the "dock_play" protocol. The docking complex was subjected to 200 steps of steepest descent minimization using Discovery Studio $4.1[22]$.

\section{Statistical Analysis}

For statistical analysis, at least three independent cell samples were included. We performed statistical tests using GraphPad InStat software (ver. 3.05; GraphPad Software, USA). We considered values as significant at ${ }^{*} p<0.05,{ }^{* *} p<0.005$, and ${ }^{* * *} p<0.001$.

\section{Results}

\section{Antibacterial Activity}

The antibacterial activity of PapN was measured by determining the MIC against eight drug-resistant bacteria. The MICs for parent papiliocin and melittin peptides were also determined. Similar to papiliocin, PapN showed potent antibacterial activity against all gram-negative bacteria tested (Table 1). The antibacterial activity of PapN against gram-negative bacteria was comparable to that of melittin, an AMP with high antibacterial activity. However, PapN and papiliocin showed a much less potent antibacterial activity against gram-positive bacteria compared with melittin. Our results showed that PapN has high selectivity against gram-negative bacteria. We further investigated the mechanism of this activity using fluorescence experiments.

\section{Cytotoxicity against Mammalian Cells}

The cytotoxicity of the peptide was tested against
NIH3T3 cells. The effects on cell survival were measured by the mitochondrial conversion of MTT to a colored formazan product, as shown in Fig. 1. PapN did not produce any cytotoxicity against NIH3T3 cells even at 100 $\mu \mathrm{M}$, whereas the control peptide, melittin, was highly toxic even at concentrations below $10 \mu \mathrm{M}$. Papiliocin resulted in a survival rate of $62 \%$ at $100 \mu \mathrm{M}$.

\section{Anti-Inflammatory Activities of PapN}

LPS, commonly referred to as an endotoxin, is a major component of the outer membrane of gram-negative bacteria. Release of LPS induces NO production during septic shock. To test the potential anti-inflammatory activity of PapN and papiliocin, we measured their abilities to inhibit NO production in LPS-stimulated RAW264.7 cells. (Fig. 2A). Papiliocin exhibited much higher inhibition of NO production than PapN. However, treatment with PapN also showed a strong, dose-dependent inhibition of NO production. At $20 \mu \mathrm{M}$, PapN inhibited about $80 \%$ NO production compared with the control.

Inhibition of the production of the cytokine TNF- $\alpha$ in LPS-stimulated cells by PapN was investigated. PapN

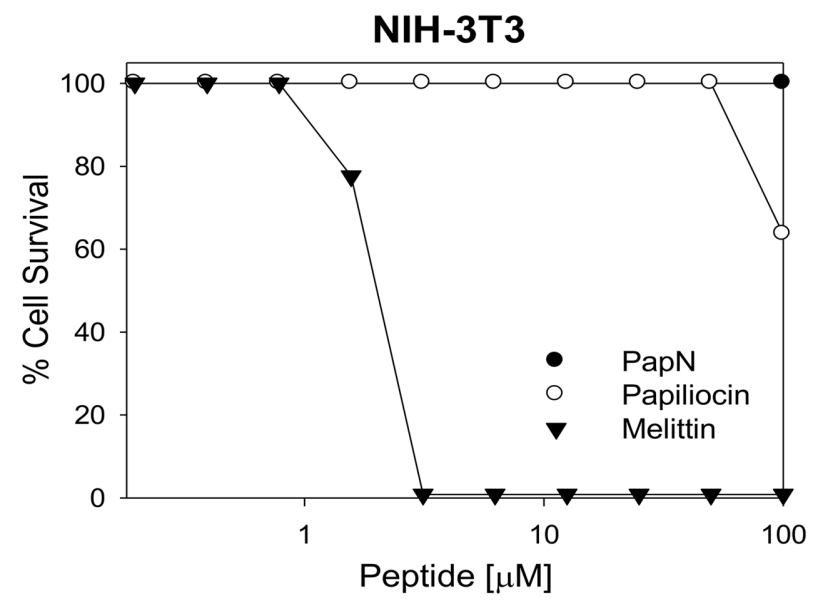

Fig. 1. Dose-dependent response to the cytotoxic activity of PapN, papiliocin, and melittin in NIH3T3 cells. 

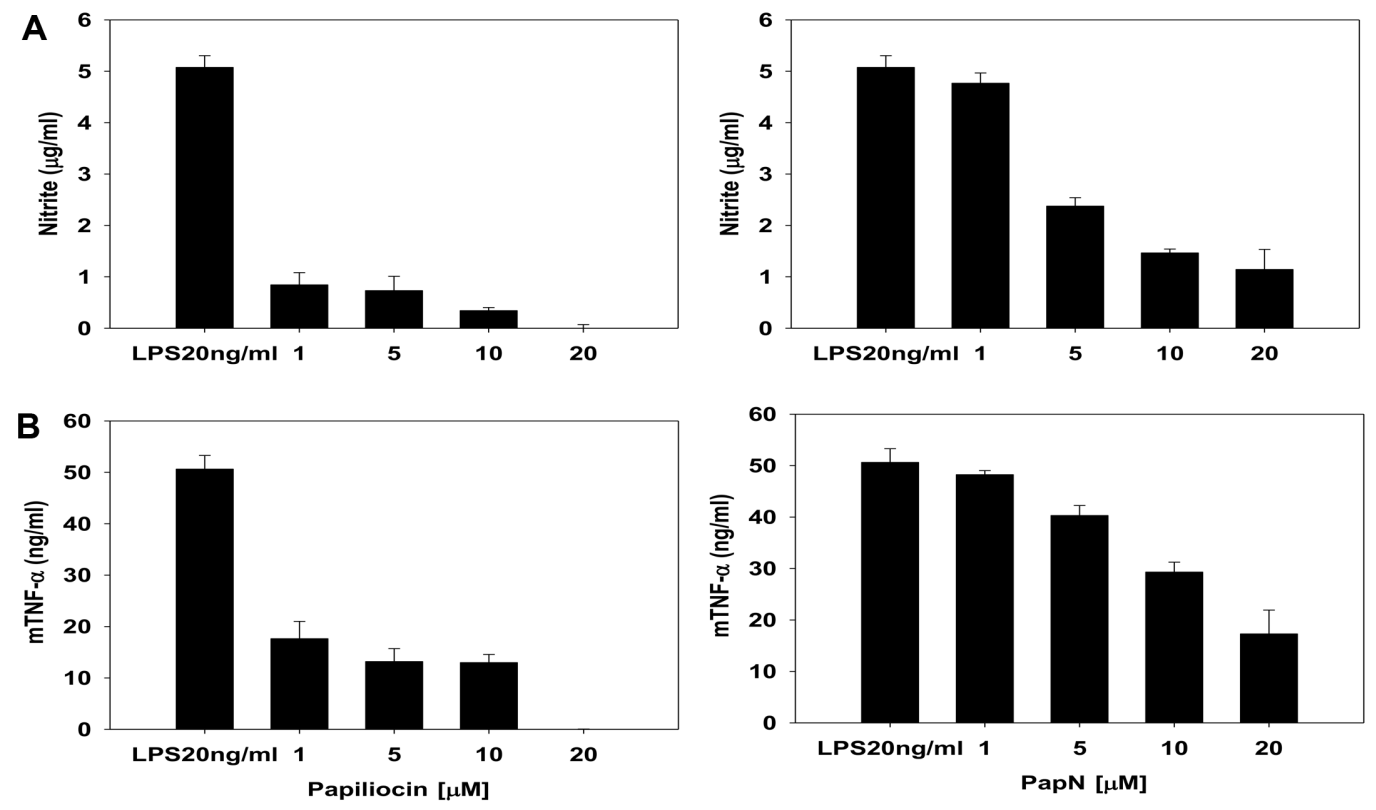

Fig. 2. Anti-inflammatory activities of PapN.

(A) Inhibition of nitrite production by papiliocin and PapN in lipopolysaccharide (LPS)-stimulated (20 ng/ml) RAW264.7 cells. (B) Inhibition of TNF- $\alpha$ production by papiliocin and PapN. Peptide concentrations from 1 to $20 \mu \mathrm{M}$ were tested.

showed potent inhibition of TNF- $\alpha$ production (Fig. 2B), and at $20 \mu \mathrm{M}$, PapN inhibited cytokine production by about $70 \%$. Compared with PapN, papiliocin showed a much higher inhibition of NO production as well as TNF- $\alpha$ production. However, treatment with PapN also resulted in a strong, dose-dependent inhibition of NO and TNF- $\alpha$ production, proving that $\mathrm{PapN}$ is a potent anti-inflammatory peptide. These data imply that the C-terminal helix of papiliocin may play an improved effect with the $\mathrm{N}$ terminal helix with respect to inhibiting $\mathrm{NO}$ and cytokine production, resulting in the high anti-inflammatory activity of papiliocin. Thus, the N-terminal helix PapN is uniquely important for the papiliocin's antibacterial activity, while also contributing to its anti-inflammatory properties.

\section{Tryptophan Fluorescence Experiments}

To investigate the interactions between tryptophan in the peptides and the gram-negative bacterial cell membrane, we monitored fluorescence emissions of Trp2 in papiliocin and PapN in SUVs composed of negatively charged phospholipids or in LPS micelles. As the vesicles and LPS were dissolved in Tris and phosphate buffers, respectively, the results were compared separately with each buffer. Papiliocin and PapN showed a blue shift greater than $18 \mathrm{~nm}$ and $16 \mathrm{~nm}$, respectively, in the presence of LPS. In comparison, they exhibited $11 \mathrm{~nm}$ and $12 \mathrm{~nm}$ blue shifts, respectively, in the presence of SUVs (Table 2). The large blue shift in LPS micelles suggested a close interaction with LPS in gram-negative bacteria.

To determine the extent to which Trp2 was buried in the gram-negative bacterial cell membrane, a fluorescence quenching experiment was performed with acrylamide as a quencher. This allowed the calculation of the Ksv for the quenching of Trp2 fluorescence by acrylamide in the presence of LPS (Table 3). The quenching of Trp2 fluorescence was equally effective with PapN as with papiliocin, suggesting that Trp2 of PapN and papiliocin was buried extensively within the negatively charged LPS, thus explaining the high inhibition of NO and cytokine production in LPS-stimulated RAW264.7 cells.

Table 2. Tryptophan fluorescence blue shift for papiliocin and PapN in various environments.

\begin{tabular}{lcccc}
\hline Peptides & $\lambda_{\max }(\mathrm{nm})$ in Phosphate buffer $(\mathrm{pH}$ 6.0) & $\lambda_{\max }(\mathrm{nm})$ in $8.0 \mu$ M LPS & $\lambda_{\max }(\mathrm{nm})$ in Tris buffer & $\lambda_{\max }(\mathrm{nm})$ in EYPC/EYPG (7:3) \\
\hline Papiliocin & 353 & 335 & 351 & 340 \\
PapN & 354 & 338 & 351 & 339 \\
\hline
\end{tabular}


Table 3. Quenching of tryptophan fluorescence in papiliocin and PapN by acrylamide.

\begin{tabular}{lcccc}
\hline \multirow{2}{*}{ Composition } & \multicolumn{2}{c}{ Papiliocin } & \multicolumn{2}{c}{ PapN } \\
\cline { 2 - 5 } & $\lambda_{\max }$ & $K_{\mathrm{sv}}$ & $\lambda_{\max }$ & $K_{\mathrm{sv}}$ \\
\hline Phosphate buffer (pH 6.0) & 352 & 30.46 & 354 & 29.25 \\
LPS & 335 & 7.39 & 338 & 7.92 \\
\hline
\end{tabular}

\section{Experiments}

The secondary structure of PapN in LPS was investigated using CD spectroscopy. As shown in Fig. 3, in aqueous solution, PapN displayed a random structure. However, upon addition of LPS, the $\alpha$-helical content of the peptide gradually increased in a dose-dependent manner with concentrations of up to $0.4 \mathrm{mg} / \mathrm{ml}$ LPS, implying that PapN acquired a more folded structure in the presence of LPS, which was used to mimic the gram-negative bacterial cell membrane environment.

\section{Binding Model of LPS and PapN}

Since CD data as well as fluorescence data proved that PapN interacts strongly with LPS and this interaction is necessary for its antibacterial and anti-inflammatory activities, we built a binding model of LPS and PapN. LPS consists of three main components: O-antigen, core oligosaccharide, and lipid A (Fig. 4A). The highly conserved lipid A portion with six lipid chains in LPS is key for inflammatory responses. Lipid A contains 3-hydroxytetradecanoic acid, myristic acid, lauric acid, phosphate ion, and 2-amino-2-deoxy-alpha-D-glucopyranose. Fig. 4B depicts papiliocin being composed of 37 amino acid residues with an amphipathic $\alpha$-helical structure at the $\mathrm{N}$-terminus and a hydrophobic C-terminal $\alpha$-helix linked by a hinge region [6]. The docking pose of LPS and PapN showed that PapN interacts with LPS through the helix region of PapN (Figs. 4C and 4D). Fig. 4C shows the predicted binding pose, where LPS and PapN are displayed as spheres and surface, respectively. The hydrophobic surface and hydrophilic surface of PapN are shown as red and blue colors, respectively. In Fig. 4D, the PapN amphipathic helix can also be seen as a cartoon, and the residues interacting with LPS are shown as sticks. Our docking model suggested that LPS may exhibit several hydrophobic and electrostatic interactions with PapN (Figs. 4C and 4D). A previous timekilling analysis against $E$. coli as well as a transmembrane potential depolarization assay with $E$. coli revealed that papiliocin completely killed $E$. coli much faster and depolarized its membrane more effectively than papiliocin W2A or papiliocin F5A mutants [7]. Accordingly, Trp2 is

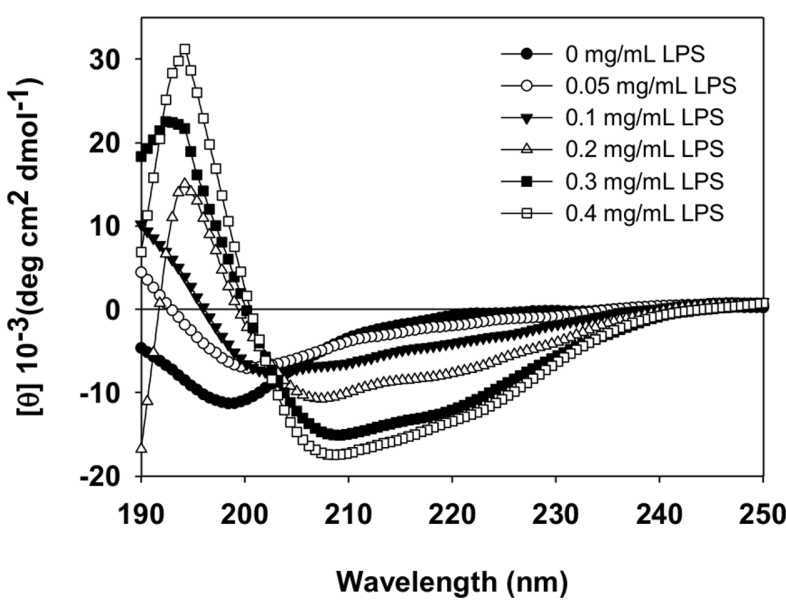

Fig. 3. CD spectra of PapN in the presence of $0-0.4 \mathrm{mg} / \mathrm{ml}$ LPS.

important for the antibacterial and anti-inflammatory activities of papiliocin [7]. Notably, in our binding model, Trp2 in PapN participated in hydrophobic interactions with the R2 lipid chain of LPS present in 3-hydroxytetradecanoic acid, akin to those of Phe5 with the R2" and R3 lipid chain of lauric acid and 3-hydroxy-tetradecanoic acid in lipid A (Fig. 4D). Furthermore, the binding model showed that the positively charged Arg1, Arg13, and Arg16 residues in the amphipathic N-terminal helix of papiliocin interacted electrostatically with the phosphate groups of lipid A in LPS (Fig. 4D). Positively charged side chains containing nitrogen atoms of Arg1, Arg13, and Arg16 shared electrostatic interactions with the phosphate of lipid A in LPS. The side-chain nitrogen atoms of Arg1 form hydrogen bond interactions with oxygen atoms in 2amino-2-deoxy-alpha-D-glucopyranose and 3-hydroxytetradecanoic acid of lipid A. Likewise, the side-chain oxygen atom of Glu9 and the nitrogen atom in 2-amino-2deoxy-alpha-D-glucopyranose of lipid A participated in hydrogen bond interactions.

\section{Discussion}

In NMR solution structure, papiliocin consists of an amphipathic $\alpha$-helix from Lys3 to Lys21 linked by a hinge region to a hydrophobic $\alpha$-helix from Ala25 to Val36 [6]. Papiliocin has a high net charge of +8 and contains seven Lys residues and three Arg residues. Notably, PapN alone possesses six Lys as well as three Arg residues, with a high net charge of +7 . Given that PapN has a positively charged amphipathic $\alpha$-helical structure, it retains the antibacterial and anti-inflammatory activities of papiliocin without cytotoxicity. This amphipathicity of PapN is critical for its 


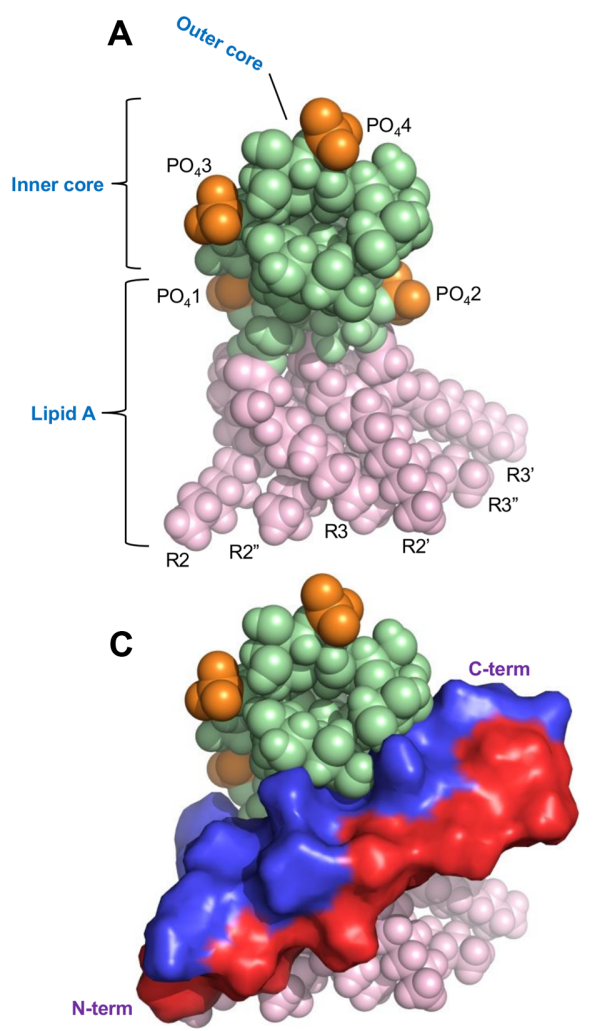

B
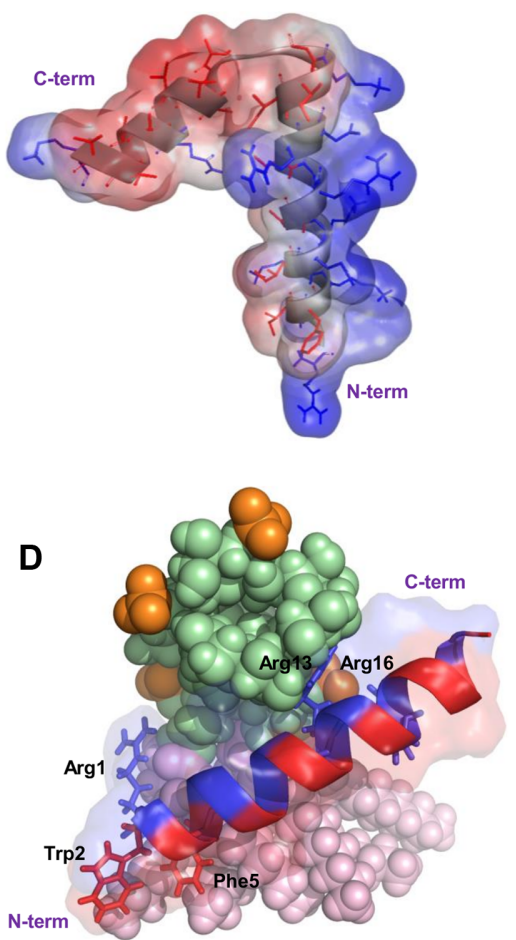

Fig. 4. Binding model of PapN to LPS.

(A) Three-dimensional structure of LPS shown as spheres. Fatty acid chains, phosphate groups, and sugar portions are shown as light pink, orange, and green colors, respectively. The region of lipid A, inner core, and outer core are shown. (B) Structure of papiliocin. The hydrophobic surface and hydrophilic surface of papiliocin are shown as red and blue colors, respectively. (C) Molecular docking pose of PapN and LPS. Spheres and surface are used to display LPS and PapN, respectively. The hydrophobic surface and hydrophilic surface of PapN are shown as red and blue colors, respectively. (D) Docking model of PapN bound to LPS. The PapN amphipathic helix is shown as a cartoon, and the residues interacting with LPS are shown as sticks. Hydrophobic and hydrophilic residues in the amphipathic helix of PapN are shown in red and blue colors, respectively.

antibacterial activity because the hydrophilic sector faces the negatively charged head groups of the phospholipid membrane, and the hydrophobic aromatic rings of Trp2 can penetrate into the acyl chains of hydrophobic lipids [8]. Furthermore, PapN exhibited potent antibacterial activities against various gram-negative multidrug-resistant bacteria, and significantly inhibited $\mathrm{NO}$ and cytokine production in LPS-stimulated RAW264.7 cells.

The main component of the gram-negative bacterial cell wall is LPS, which consists of a lipid A moiety and repeating polysaccharide moieties. LPS acts as a strong stimulator of macrophages and dendritic cells, which are part of the innate immunity of various organisms [11]. LPS covers the outer membrane of the gram-negative bacterial cell wall and triggers the secretion of inflammatory cytokines, resulting in septic shock and other immune diseases. The LPS-binding protein carries LPS and presents it to the accessory protein called cluster of differentiation 14, which participates in TLR4 signaling after the active complex TLR4/MD-2/LPS is formed [23]. TLR4 signaling leads to proinflammatory cytokines that are important to activate potent immune responses [23]. However, uncontrolled inflammatory responses induced by LPS through TLR4 may lead to autoimmune diseases such as sepsis atherosclerosis, asthma, ischemia/reperfusion, and rheumatoid arthritis that can cause organ failure or even death, and thus anti-inflammatory activity is a vital process [24, 25]. Various biophysical experiments have investigated the interactions between AMPs and LPS [26, 27]. In this work, we used tryptophan fluorescence and CD spectroscopy to study the folding and the interactions between the Trp2 residue of PapN and LPS. The quenching of Trp2 fluorescence in PapN was effective in the presence of LPS micelles and was comparable to that of papiliocin. Trp2 at the N-terminus 
appeared to be buried deeply inside the negatively charged LPS and, based on the large blue shift in the presence of LPS micelles, PapN was likely to interact tightly with LPS in gram-negative bacteria. Spectroscopic experiments involving $\mathrm{CD}$ also confirmed the gradual formation of an $\alpha$-helical PapN structure upon addition of LPS, suggesting that this interaction resulted in potent anti-inflammatory activity of PapN.

Assuming that the anti-inflammatory activity of PapN might be related to the direct interaction between PapN and LPS, we used a docking study to investigate the binding model between LPS and PapN. Neutralization of LPS is a significant feature of AMPs' action against sepsis. AMPs such as lactoferrin, Limulus anti-lipopolysaccharide factor, cathelicidins, polymyxin B, and granulysins are promising peptides against sepsis owing to their direct interaction with LPS in gram-negative bacteria [15, 16]. Here, we showed that the positively charged Arg1, Arg13, and Arg16 residues of PapN interacted with the negatively charged phosphate of lipid A, and Trp2 and Phe5 in PapN engaged in hydrophobic interactions with the R2, R2", and R3 acyl chain of lipid A. Docking models between LPS and AMPs such as cecropin P1, tachyplesin I, esculentin-1a(121) $\mathrm{NH}_{2}$, and esculentin-1a(1-21) $\mathrm{NH}_{2} 1 \mathrm{c}$ have been proposed [28-30].

High cationicity in the peptides that bind to LPS is required to neutralize the LPS. In our previous study, by checking the fluorescence intensity of FITC-conjugated LPS, we revealed the importance of electrostatic interactions between positively charged residues in PapN and LPS for LPS dissociation through mutation of the two key residues Trp2 and Phe5 in papiliocin [8]. Tachyplesin I (KWCFRV CYRGICYRRCR-NH2) is an AMP with six positively charged residues that can bind to LPS [30]. Moreover, NMR studies also prove that LPS interacts with Arg17, and phosphate groups of LPS may possibly bind to tachyplesin I. One docking model suggests that Arg14, Arg15, Arg17, and Lys1 participate in electrostatic interactions with LPS [30]. Similarly, Trp2 and Phe4 make hydrophobic interactions with the fatty acyl chains of LPS [30]. Cecropin P1 (SWL SKTAKKLENSAKKRISEGIAIAIQGGPR) is another AMP that has been proven to bind with LPS [28]. The docking model shows that the residues Lys15 and Lys16 may interact with phosphate, and Ile22 and Ile26 may bind with lipid chains of LPS [28]. Esculentin-1a(1-21) $\mathrm{NH}_{2}$ (GIFSKLAGK KIKNLLISGLKG-NH ) is the other peptide that may bind to LPS through Lys5, Lys9, Lys12, and Lys20 [31]. Similarly, esculentin-1a(1-21) $\mathrm{NH}_{2}$ 1c (GIFSKLAGKKIKN ${ }^{\mathrm{d}}$ LLI $^{\mathrm{d}}$ SGLKG$\mathrm{NH}_{2}$ ) can bind with LPS through Lys5 an Lys10. NMR studies suggest that these two peptides interact with the phosphate groups and fatty acyl chains of LPS through electrostatic and hydrophobic interactions, respectively [29]. Lauryl-LF11 peptide had antimicrobial activity against Salmonella enterica gram-negative bacteria, and electrostatic interactions were proven important for the peptide-LPS binding [8].

Similar to our docking pose, all those predicted poses displayed significant interactions between positively charged residues of peptide and negatively charged phosphate groups in the polar head of LPS. In addition, our model exhibited hydrophobic interactions between nonpolar fatty acid tails in LPS and hydrophobic residues of PapN. Hence, our molecular docking pose agrees with the other proposed binding modes between peptide and LPS.

In conclusion, PapN presents high antibacterial and antiinflammatory activities, but without cytotoxicity. Importantly, PapN interacts strongly with LPS through Trp2 and Phe5 as well as positively charged Arg1, Arg13, and Arg16 in the amphipathic $\alpha$-helical structure of PapN. These properties make PapN a potent peptide antibiotic suitable for treating the endotoxin shock and sepsis caused by gram-negative bacterial infections. Future in vivo studies will determine the potency of PapN for treating the septic shock caused by LPS of gram-negative bacteria and will confirm the current biophysical results as well as structure-function relationships of PapN.

\section{Acknowledgments}

This work was supported by a grant from the National Research Foundation of Korea (NRF) funded by the Korean Government (MSIT) (2016R1A2B2008543). P. Durai was supported partly by the 2017 Brain Pool program of Konkuk University.

\section{Conflict of Interest}

The authors have no financial conflicts of interest to declare.

\section{References}

1. Hancock REW, Nijnik A, Philpott DJ. 2012. Modulating immunity as a therapy for bacterial infections. Nat. Rev. Microbiol. 10: 243-254.

2. Haney EF, Mansour SC, Hancock REW. 2017. Antimicrobial peptides: an introduction, pp. 3-22. In Hansen $\mathrm{P}$ (ed.). Antimicrobial Peptides. Methods in Molecular Biology, Vol. 1548. Humana Press, New York. 
3. Koehbach J. 2017. Structure-activity relationships of insect defensins. Front. Chem. 5: 45.

4. Yi HY, Chowdhury M, Huang YD, Yu XQ. 2014. Insect antimicrobial peptides and their applications. Appl. Microbiol. Biotechnol. 98: 5807-5822.

5. Kim SR, Hong MY, Park SW, Choi KH, Yun EY, Goo TW, et al. 2010. Characterization and cDNA cloning of a cecropinlike antimicrobial peptide, papiliocin, from the swallowtail butterfly, Papilio xuthus. Mol. Cells 29: 419-423.

6. Kim JK, Lee E, Shin S, Jeong KW, Lee JY, Bae SY, et al. 2011. Structure and function of papiliocin with antimicrobial and anti-inflammatory activities isolated from the swallowtail butterfly, Papilio xuthus. J. Biol. Chem. 286: 41296-41311.

7. Lee J, Hwang JS, Hwang B, Kim JK, Kim SR, Kim Y, et al. 2010. Influence of the papiliocin peptide derived from Papilio xuthus on the perturbation of fungal cell membranes. FEMS Microbiol Lett. 311: 70-75.

8. Lee E, Kim JK, Jeon D, Jeong KW, Shin A, Kim Y. 2015. Functional roles of aromatic residues and helices of papiliocin in its antimicrobial and anti-inflammatory activities. Sci. Rep. 5: 12048.

9. Jeon D, Jacob D, Kwak C, Kim Y. 2017. Short antimicrobial peptides exhibiting antibacterial and anti-inflammatory activities derived from the N-terminal helix of papiliocin. Bull. Korean Chem. Soc. 38: 1260-1268.

10. Bowdish DM, Davidson DJ, Scott MG, Hancock RE. 2005. Immunomodulatory activities of small host defense peptides. Antimicrob. Agents Chemother. 49: 1727-1732.

11. Rosenfeld Y, Papo N, Shai Y. 2006. Endotoxin (lipopolysaccharide) neutralization by innate immunity host-defense peptides. Peptide properties and plausible modes of action. J. Biol. Chem. 281: 1636-1643.

12. Nell MJ, Tjabringa GS, Wafelman AR, Verrijk R, Hiemstra PS, Drijfhout JW, et al. 2006. Development of novel LL-37 derived antimicrobial peptides with LPS and LTA neutralizing and antimicrobial activities for therapeutic application. Peptides 27: 649-660.

13. Frohm M, Agerberth B, Ahangari G, Stâhle-Bäckdahl M, Lidén S, Wigzell $\mathrm{H}$, et al. 1997. The expression of the gene coding for the antibacterial peptide LL-37 is induced in human keratinocytes during inflammatory disorders. J. Biol. Chem. 272: 15258-15263.

14. Lee PH, Ohtake T, Zaiou M, Murakami M, Rudisill JA, Lin $\mathrm{KH}$, et al. 2005. Expression of an additional cathelicidin antimicrobial peptide protects against bacterial skin infection. Proc. Natl. Acad. Sci. USA 102: 3750-3755.

15. Brandenburg K, Heinbockel L, Correa W, Lohner K. 2016. Peptides with dual mode of action: killing bacteria and preventing endotoxin-induced sepsis. Biochim. Biophys. Acta 1858: 971-979.

16. Terayama T, Yamakawa K, Umemura $Y$, Aihara M, Fujimi S. 2017. Polymyxin B hemoperfusion for sepsis and septic shock: a systematic review and meta-analysis. Surg. Infect. 18: 225-233.

17. Lee E, Kim JK, Shin S, Jeong KW, Shin A, Lee J, et al. 2013. Insight into the antimicrobial activities of coprisin isolated from the dung beetle, Copris tripartitus, revealed by structureactivity relationships. Biochim. Biophys. Acta 1828: 271-283.

18. Jeon D, Jeong MC, Jacob B, Bang JK, Kim EH, Cheong C, et al. 2017. Investigation of cationicity and structure of pseudin-2 analogues for enhanced bacterial selectivity and anti-inflammatory activity. Sci. Rep. 7: 1455.

19. Green LC, Wagner DA, Glogowski J, Skipper PL, Wishnok JS, Tannenbaum SR. 1982. Analysis of nitrate, nitrite, and [15N]nitrate in biological fluids. Anal. Biochem. 126: 131-138.

20. Jnawali HN, Lee E, Jeong KW, Shin A, Heo YS, Kim Y. 2014. Anti-inflammatory activity of rhamnetin and a model of its binding to c-Jun $\mathrm{NH}_{2}$-terminal kinase 1 and p38 MAPK. J. Nat. Prod. 77: 258-263.

21. Trott O, Olson AJ. 2010. AutoDock Vina: improving the speed and accuracy of docking with a new scoring function, efficient optimization, and multithreading. J. Comput. Chem. 31: 455-461.

22. Dassault Systèmes BIOVIA. 2015. Discovery Studio, 4.1. Dassault Systèmes, San Diego.

23. Lee CC, Avalos AM, Ploegh HL. 2012. Accessory molecules for Toll-like receptors and their function. Nat. Rev. Immunol. 12: 168-179.

24. Kondo T, Kawai T, Akira S. 2012. Dissecting negative regulation of Toll-like receptor signaling. Trends Immunol. 33.9: 449-458.

25. O'Neill LA, Bryant CE, Doyle SL. 2009. Therapeutic targeting of Toll-like receptors for infectious and inflammatory diseases and cancer. Pharmacol. Rev. 61: 177-197.

26. Bhunia A, Domadia PN, Torres J, Hallock KJ, Ramamoorthy A, Bhattacharjya S. 2010. NMR structure of pardaxin, a poreforming antimicrobial peptide, in lipopolysaccharide micelles: mechanism of outer membrane permeabilization. J. Biol. Chem. 285: 3883-3895.

27. Sudheendra US, Dhople V, Datta A, Kar RK, Shelburne CE, Bhunia A, et al. 2014. Membrane disruptive antimicrobial activities of human $\beta$-defensin-3 analogs. Eur. J. Med. Chem. 91: 91-99.

28. Baek MH, Kamiya M, Kushibiki T, Nakazumi T, Tomisawa S, Abe C, et al. 2016. Lipopolysaccharide-bound structure of the antimicrobial peptide cecropin P1 determined by nuclear magnetic resonance spectroscopy. J. Pept. Sci. 22: 214-221.

29. Ghosh A, Bera S, Shai Y, Mangoni ML, Bhunia A. 2016. NMR structure and binding of esculentin- $1 \mathrm{a}(1-21) \mathrm{NH}_{2}$ and its diastereomer to lipopolysaccharide: correlation with biological functions. Biochim. Biophys. Acta 1858: 800-812.

30. Kushibiki T, Kamiya M, Aizawa T, Kumaki Y, Kikukawa T, Mizuguchi M, et al. 2014. Interaction between tachyplesin I, an antimicrobial peptide derived from horseshoe crab, and lipopolysaccharide. Biochim. Biophys. Acta 1844: 527-534. 